\title{
Deformation Calculating of Electromagnetic Launcher's Rail Subjected to Sinusoidal Magnetic Pressure
}

\author{
Liu Wen, ${ }^{1}$ Shi Lei, ${ }^{1}$ and Bai Xiangzhong ${ }^{2}$ \\ ${ }^{1}$ College of Sciences, Yanshan University, Qinhuangdao 066004, China \\ ${ }^{2}$ College of Civil Engineering and Mechanics, Yanshan University, Qinhuangdao 066004, China
}

Correspondence should be addressed to Liu Wen, liuwen1961@hotmail.com

Received 4 February 2010; Accepted 28 October 2010

Academic Editor: Marcelo Messias

Copyright (C) 2011 Liu Wen et al. This is an open access article distributed under the Creative Commons Attribution License, which permits unrestricted use, distribution, and reproduction in any medium, provided the original work is properly cited.

\begin{abstract}
The electromagnetic launcher's rail can be modeled as a beam on elastic foundation with simply supported beam by moving load. In this paper, Euler beam theory is applied to build the mechanical model, and the analytical solution of the equation subjected to sinusoidal magnetic pressure is derived in detail, which has successfully avoided the errors which are caused by using the uniform pressure to approximately replace the variable force. Numerical analysis of the influences brought from the elastic coefficient, the damping coefficient, the mass of rail, and the load's velocity on the deformation of beam by the MATLAB software show that the elastic coefficient and the load's velocity have quite obvious effect on the deformation of the beam while the damping coefficient and the mass of rail have not obvious effect on the deformation of the beam.
\end{abstract}

\section{Introduction}

As a new concept weapon, the electromagnetic railgun has adopted the electromagnetic launching method, not only broken through the limitation of projectile velocity by the traditional chemical energy, but also greatly surpassed the rate of cannon projectile. Hence, it has a huge application potential in the military field. Since the 1980s, especially in the recent ten years, with the development of new technology and new material, the research of launcher, launching weight, projectile velocity, and high efficiency power source in the electromagnetic railgun has reached a series of achievements. The Su Rense.Livermore Country Laboratory and The Lowes.Alamos Country Laboratory, once have cooperated to accelerate a projectile weighed $2.2 \mathrm{~g}$ to a supervelocity of $10 \mathrm{~km} / \mathrm{s}$. Fluid Physics Institute of 
the Chinese Engineering Academy had built the first electromagnetic rail launcher, which can accelerate the projectile weighted $0.34 \mathrm{~g}$ to $16.8 \mathrm{~km} / \mathrm{s}$ [1]. While the velocity of the conventional cannon is only $2 \mathrm{~km} / \mathrm{s}$, which is so close to the limitation of physics that the range is not possible to be farther. On the contrary, the thrust of the electromagnetic railgun is ten times bigger than that of the traditional launcher. The projectile can be accelerated to several kilometers or even to dozens of kilometers in one second, for it possesses huge kinetic energy which greatly enhance the range and power of the weapon [2-4].

Tzeng and sun [5] used the elastic foundation beam to build mechanical model of the electromagnetic railgun and deduced the solve process of governing equation. Yuwei [6] analyzed theoretical built model and simulation analysis for the work of the process of electromagnetic railgun. Sheng [7] adopted the Fourier transform to study displacement field due to a moving load on Euler beam resting on an elastic half-space.

However, the damping force to the response of beam is ignored in the above researches. There is no doubt that the calculation of this kind of situation has the defects of analysis and calculation of mechanical. As a high-tech and high-precision electromagnetic launcher, accurate theoretical analysis and calculation in engineering are require. But until now, no researcher has given any exact analytical solution; thus, further analytic solution of the equation subjected to variable pressure is of great significance. In fact, it is of theoretical value to research the theoretical analytic solution of various disciplines, including the analytical solution of practical engineering problems. On the one hand, its mechanical picture can be completely stated; on the other hand, it can be used as a standard solution to widely produce variety of numerical solution.

In this paper, regarding the rail as simply supported beam on the elastic foundation and considering the damping force, a mechanic model which is under the effect of moving load is proposed. Moreover, making use of variable method and the Lagrange equation considering the damping force, the analytical solution $[8,9]$ of the governing equation subjected to nonlinear function pressures is derived and the influences brought from the elastic coefficient, the damping coefficient, the mass of rail, and the load's velocity on the response of beam are analyzed.

\section{Mechanical Model}

Figure 1 shows a schematic of an electromagnetic railgun composed of power source, rail, armature, and projectile. When the electric current of armature goes through the rail, it forms a strong magnetic field in the area of their encirclement. With the reaction by the magnetic field and the electric current, it emerges powerful electromagnetic force, which pushes the armature and projectile to do the accelerating motion along the rail till the projectile is launched out of the rail.

Figure 2 is the mechanical model of the railgun, simply supported beam partially subject to nonlinear load in a time-varying region sitting on the elastic foundation. Considering the effect of the beam by the damping force and basing on the Euler beam theory, we obtain the governing equation of elastic foundation beam by moving load which is a transient fourth-order differential equation as follows $[10,11]$ :

$$
m \frac{\partial^{2} w}{\partial t^{2}}+E I \frac{\partial^{4} w}{\partial x^{4}}+k w+c \frac{\partial w}{\partial t}=p(x, t)
$$




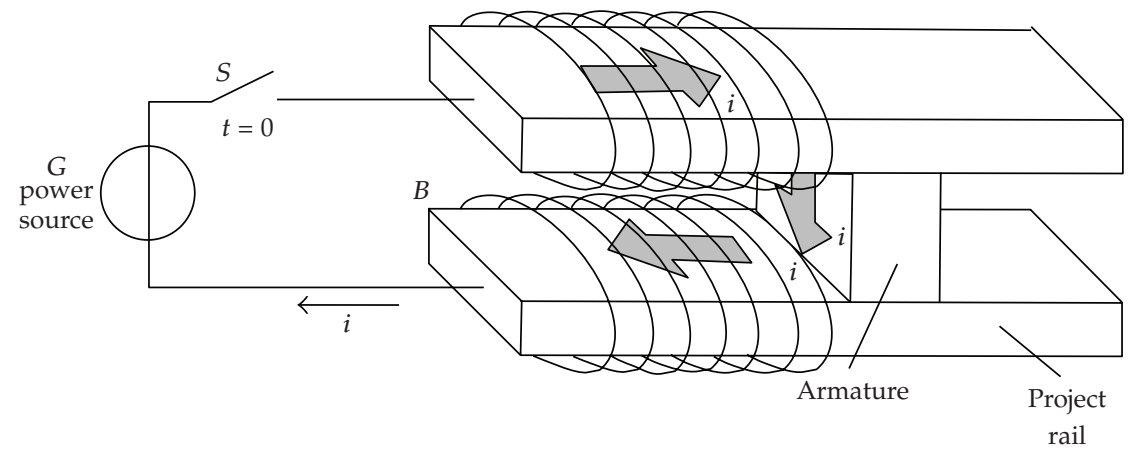

Figure 1: The general diagram of the railgun.

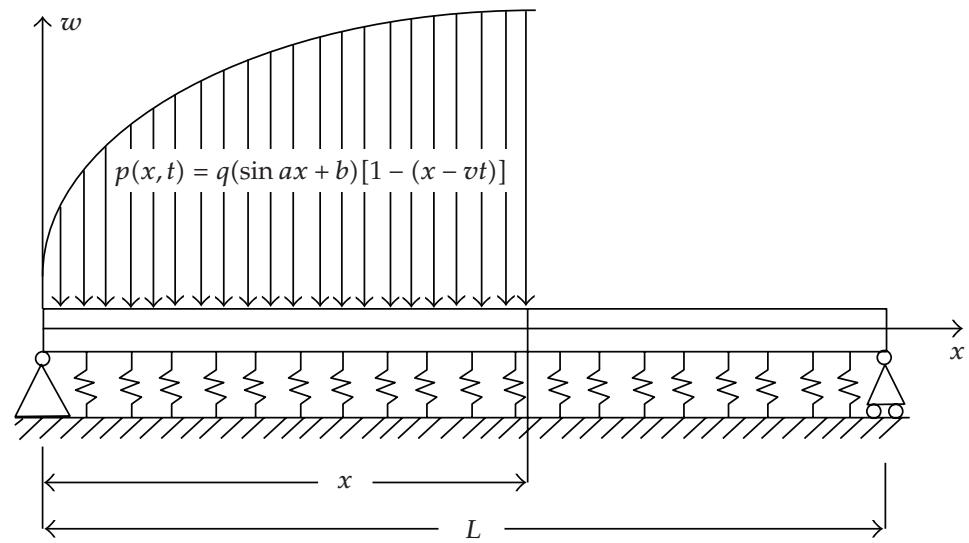

Figure 2: The rail is modeled as a beam on elastic foundation.

where $w$ is the deflection, $m=\rho B h$ is the mass per unit length, $\rho$ is the density of rail material, $B$ and $h$ are, respectively, the width and thickness of the rail, $E I$ is the bending stiffness of beam, $k$ is the elastic constant, and $c$ is the damping coefficient. We have the function $p(x, t)=$ $q(x)[1-H(x-v t)]$ In (2.1), represents the magnetic pressure front traveling along the rail with velocity $v$ represented by a Heaviside step function $H(x-v t)$ [12], and $q(x)=q(\sin a x+b)$.

\section{Solution of the Homogeneous Equation}

The homogeneous equation is a fourth-order partial differential equation, and in order to change it into the ordinary differential equation, we solve it by the method of variable separation.

The solution of the homogeneous equation of (2.1) can be expressed as follows:

$$
w(x, t)=\phi(t) \theta(x) .
$$

Substituting (3.1) into the homogeneous equation (2.1): 


$$
m \frac{\partial^{2} \phi}{\partial t^{2}} \theta+E I \phi \theta^{(4)}+k \phi \theta+c \theta \frac{\partial \phi}{\partial t}=0 .
$$

That can be expressed as follows:

$$
-\frac{\partial^{2} \phi / \partial t^{2}}{c \phi}-\frac{\partial \phi / \partial t}{m \phi}=\frac{E I}{c m} \frac{\theta^{(4)}}{\theta}+\frac{k}{c m} .
$$

From (3.3), let

$$
\begin{gathered}
-\frac{\partial^{2} \phi / \partial t^{2}}{c \phi}-\frac{\partial \phi / \partial t}{m \phi}=\lambda^{2}, \\
\frac{E I}{c m} \frac{\theta^{(4)}}{\theta}+\frac{k}{c m}=\lambda^{2} .
\end{gathered}
$$

That is,

$$
\theta^{(4)}-\beta^{4} \theta=0,
$$

where

$$
\beta^{4}=\left(\lambda^{2}-\frac{k}{c m}\right) \frac{c m}{E I}
$$

Solution of (3.4) can be expressed as follows:

$$
\phi(t)=A e^{((-c+R) / 2 m) t}+B e^{((-c-R) / 2 m) t},
$$

where

$$
R^{2}=c^{2}-4 c m^{2} \lambda^{2}>0 .
$$

Based on the boundary condition of the simple beam,

$$
\begin{gathered}
\theta(0)=0, \\
\left.\frac{\partial^{2} \theta(x)}{\partial x^{2}}\right|_{x=0}=0, \\
\theta(L)=0, \\
\left.\frac{\partial^{2} \theta(x)}{\partial x^{2}}\right|_{x=L}=0 .
\end{gathered}
$$


Solution of (3.6) can be expressed as follows [13]:

$$
\theta_{i}(x)=\sqrt{\frac{2}{m L}} \sin \frac{n \pi}{L} x
$$

Accordingly:

$$
w_{i}(x, t)=\theta_{i}(x) \phi_{i}(t)=\left(\sqrt{\frac{2}{m L}} \sin \frac{n \pi}{L} x\right) \times\left[A_{i} e^{((-c+R) / 2 m) t}+B_{i} e^{((-c-R) / 2 m) t}\right] .
$$

In terms of the orthogonality of $\theta_{i}(x)$ [14], we obtain

$$
\int_{0}^{L} \theta_{i} \theta_{j} d x= \begin{cases}0, & \text { at } i \neq j \\ 1, & \text { at } i=j\end{cases}
$$

Hence, deformation $w(x, t)$ of the beam can be expressed by the linear combination of $\theta_{i}(x)$

$$
w(x, t)=\sum_{i} \theta_{i}(x) \phi_{i}(t)=\sum_{i}\left(\sqrt{\frac{2}{m L}} \sin \frac{n \pi}{L} x\right) \times\left[A_{i} e^{((-c+R) / 2 m) t}+B_{i} e^{((-c-R) / 2 m) t}\right],
$$

where constants $A_{i}, B_{i}$ are determined by the initial conditions.

\section{Analytical Solution of Governing Equation}

The analytical solution of (2.1) can be obtained by the Lagrange equation including the damping force, where $T$ is the kinetic energy of the beam, $U$ is the total stain energy, and $G$ is the dissipation function [15]. We have

$$
\frac{d}{d t}\left(\frac{\partial T}{\partial \phi_{i} / \partial t}\right)-\frac{\partial T}{\partial \phi_{i}}+\frac{\partial U}{\partial \phi_{i}}-\frac{\partial G}{\partial \phi_{i} / \partial t}=Q_{i}
$$

The kinetic energy of the beam $T$ can be expressed as follows [16]:

$$
T=\frac{1}{2} \sum_{i} M_{i}\left(\frac{\partial \phi_{i}}{\partial t}\right)^{2}
$$

where $M_{i}=\int_{0}^{L} m \theta_{i}^{2}(x) d x$ represents the general mass of the beam. 
The total strain energy of the beam $U$ is consisted of the strain energy $U_{b}$ of the beam and the strain energy $U_{f}$ of the foundation

$$
\begin{aligned}
U_{b} & =\frac{1}{2} \int_{0}^{L} E I\left(\frac{\partial^{2} w(x, t)}{\partial x^{2}}\right)^{2} d x \\
& =\frac{1}{2} \sum_{i} \sum_{j} \phi_{i} \phi_{j} \int_{0}^{L} E I \frac{\partial^{2} \theta_{i}}{\partial x^{2}} \frac{\partial^{2} \theta_{j}}{\partial x^{2}} d x \\
& =\frac{1}{2} \frac{E I}{m} \sum_{i} \beta_{i}^{4} M_{i} \phi_{i}^{2}, \\
U_{f} & =\frac{1}{2} \int_{0}^{L} k w^{2} d x=\frac{k}{2} \int_{0}^{L}\left(\sum_{i} \theta_{i} \varphi_{i}\right)^{2} d x \\
& =\frac{k}{2} \sum_{i} \sum_{j} \phi_{i} \phi_{j} \int_{0}^{L} \theta_{i} \theta_{j} d x \\
& =\frac{k}{2 m} M_{i} \sum_{i} \phi_{i}^{2} .
\end{aligned}
$$

The total strain energy $U$ is obtained as

$$
\begin{aligned}
U & =U_{b}+U_{f}=\frac{1}{2} \frac{E I}{m} \sum_{i} \beta_{i}^{4} M_{i} \phi_{i}^{2}+\frac{k}{2 m} M_{i} \sum_{i} \phi_{i}^{2} \\
& =\frac{1}{2}\left[\sum_{i} M_{i} \phi_{i}^{2}\left(\frac{E I}{m} \beta_{i}^{4}+\frac{k}{m}\right)\right] \\
& =\frac{1}{2}\left[\sum_{i} c M_{i} \phi_{i}^{2} \lambda_{i}^{2}\right] .
\end{aligned}
$$

The dissipation function $G$ can be expressed as

$$
\begin{aligned}
G & =-\frac{1}{2} \int_{0}^{L} c\left(\frac{\partial w}{\partial t}\right)^{2} d x \\
& =-\frac{1}{2} \sum_{i} \sum_{j} \frac{\partial \phi_{i}}{\partial t} \frac{\partial \phi_{j}}{\partial t} \int_{0}^{L} c \theta_{i} \theta_{j} d x \\
& =-\frac{c}{2 m} \sum_{i} M_{i}\left(\frac{\partial \phi_{i}}{\partial t}\right)^{2} .
\end{aligned}
$$

The virtual work done by the magnetic pressure $p(x, t)=q(x)[1-H(x-v t)]$ in a virtual displacement $\delta \varphi_{i}$ can be expressed as follows: 
Mathematical Problems in Engineering

$$
\begin{aligned}
\partial W & =\int_{0}^{L} p(x, t) \delta w_{i} d x \\
& =\sum_{i} \partial \phi_{i} \int_{0}^{L} p(x, t) \theta_{i}(x) d x \\
& =\sum_{i} \partial \phi_{i} Q_{i}
\end{aligned}
$$

where we define $Q_{i}$ as the generalized force

$$
\begin{aligned}
Q_{i}= & \int_{0}^{L} p(x, t) \theta_{i}(x) d x=\int_{0}^{v t} q(x) \theta_{i}(x) d x \\
= & \sqrt{\frac{2}{m L}} \int_{0}^{v t} q(\sin a x+b) \sin \frac{n \pi x}{L} d x \\
= & \frac{q L(a L+n \pi)}{(a L-n \pi)} \sqrt{\frac{1}{2 m L}}\left((a L+n \pi) \sin \left(v t \frac{a L-n \pi}{L}\right)-(a L-n \pi) \sin \left(v t \frac{a L+n \pi}{L}\right)\right) \\
& -\sqrt{\frac{2 L}{m}} \frac{b q}{n \pi}\left(\cos \frac{n \pi v t}{L}-1\right) .
\end{aligned}
$$

Substituting $T, U, G, Q_{i}$ into the Lagrange equation including the damping force, we obtain an ordinary differential equation:

$$
\frac{\partial^{2} \phi_{i}}{\partial t^{2}}+\frac{c}{m} \frac{\partial \phi}{\partial t}+c \lambda_{i}^{2} \phi_{i}=\frac{Q_{i}(t)}{M_{i}}=F(t)
$$

where

$$
\begin{aligned}
F(t)= & \frac{q L(a L+n \pi)}{M_{i}(a L-n \pi)} \sqrt{\frac{1}{2 m L}}\left((a L+n \pi) \sin \left(v t \frac{a L-n \pi}{L}\right)-(a L-n \pi) \sin \left(v t \frac{a L+n \pi}{L}\right)\right) \\
& -\sqrt{\frac{2 L}{m}} \frac{b q}{n \pi M_{i}}\left(\cos \frac{n \pi v t}{L}-1\right) .
\end{aligned}
$$

The general solution of $(4.8)$ is

$$
\begin{aligned}
\phi_{i}(t)= & \frac{m}{R}\left(\frac{\partial \phi_{i}(0)}{\partial t}+\frac{c+R}{2 m} \phi_{i}(0)\right) e^{((-c+R) / 2 m) t}+\frac{m}{R}\left(-\frac{\partial \phi_{i}(0)}{\partial t}+\frac{-c+R}{2 m} \phi_{i}(0)\right) e^{((-c-R) / 2 m) t} \\
& +\frac{m}{R} \int_{0}^{t} F(\xi)\left(e^{((-c+R) / 2 m)(t-\xi)}-e^{((-c-R) / 2 m)(t-\xi)}\right) d \xi .
\end{aligned}
$$


So, the general solution of (2.1) can be expressed as follows:

$$
\begin{aligned}
w(x, t)=\sum_{i} \theta_{i}(x) \phi_{i}(t)=\sum_{i} \sqrt{\frac{2}{m L}} \sin \frac{n \pi}{L} x \\
\times\left\{\frac{m}{R}\left(\frac{\partial \phi_{i}(0)}{\partial t}+\frac{c+R}{2 m} \phi_{i}(0)\right) e^{((-c+R) / 2 m) t}\right. \\
+\frac{m}{R}\left(-\frac{\partial \phi_{i}(0)}{\partial t}+\frac{-c+R}{2 m} \phi_{i}(0)\right) e^{((-c-R) / 2 m) t} \\
\left.+\frac{m}{R} \int_{0}^{t} F(\xi)\left(e^{((-c+R) / 2 m)(t-\xi)}-e^{((-c-R) / 2 m)(t-\xi)}\right) d \xi\right\} .
\end{aligned}
$$

The initial conditions are

$$
\phi(0)=0,\left.\quad \frac{\partial \phi(t)}{\partial t}\right|_{t=0}=0
$$

So,

$$
\phi_{i}(t)=\frac{m}{R} \int_{0}^{v \xi} F(\xi)\left(e^{((-c+R) / 2 m)(t-\xi)}-e^{((-c-R) / 2 m)(t-\xi)}\right) d \xi
$$

According, substituting the solution of (4.13) into (4.11), we can get the solution $w(x, t)$ of $(2.1)$. The moment and the shear force of the beam in the rail can be further derived from $w(x, t)$, which provides basis for the overall investigation of the dynamic behavior of the electromagnetic railgun.

\section{Numerical Analysis}

Since there are differences among materials of electromagnetic rail launcher, the damping force and the rate of moving load will possibly bring influence to the response of the rail [17]. Thus, it is necessary to consider the elastic coefficient, the damping coefficient, the mass of rail, and the load's velocity to compare the response of the rail.

A known Material is modulus of rail material $E=120 \mathrm{GPa}$, the elastic constant $k=$ $2.532 \times 10^{10} \mathrm{~N} / \mathrm{m}^{2}$, the density of rail material $\rho=8700 \mathrm{~kg} / \mathrm{m}^{3}$, the width of rail $B=3 \times 10^{-2} \mathrm{~m}$, the thickness of rail $h=1 \times 10^{-2} \mathrm{~m}$, the length of rail $L=2 \mathrm{~m}$, and the magnetic load collection degree $q(x)=110 \sin x \mathrm{MPa}[18]$.

Figure 3 shows the deformation of the beam by the elastic coefficient. Along with the elastic coefficient $(k)$ increasing, the curve of time deformation is a decreasing trend. Under the calculating conditions given by this paper, for the rail of which $k$ equals to $2.532 \times 10^{10} \mathrm{~N} / \mathrm{m}^{2}$, the deformation $(w)$ of the beam is $1 \times 10^{-3} \mathrm{~m}$ when the armature moves to the moment $t=1 \times 10^{-3}$. While for the rail of which $k$ equals to $5.064 \times 10^{10} \mathrm{~N} / \mathrm{m}^{3}$, the deformation $(w)$ of the beam is $0.45 \times 10^{-3} \mathrm{~m}$ at the same moment, and we can see that the former is $122 \%$ bigger than the latter. 


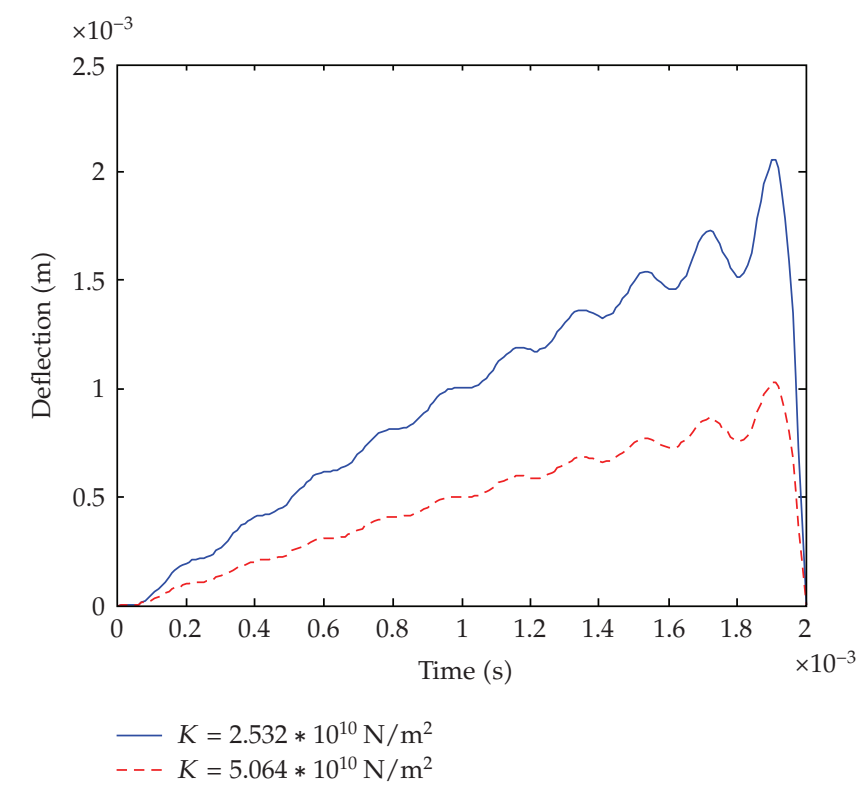

Figure 3: Deformation curve by differences elastic coefficient.

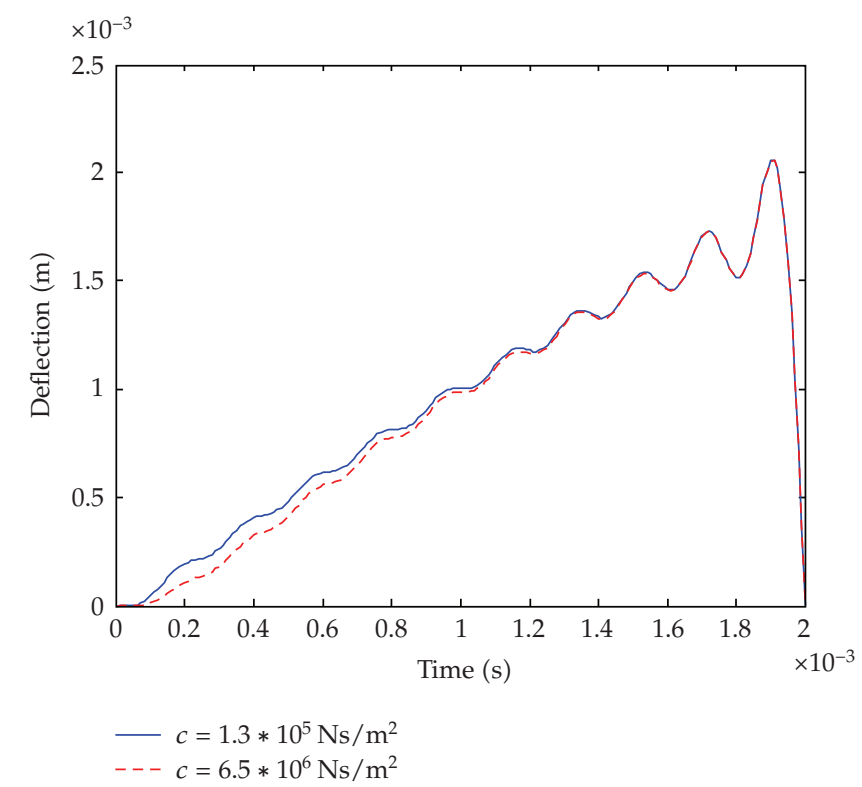

Figure 4: Deformation curve by differences damping coefficient.

Figure 4 shows the deformation of the beam by the damping coefficient. Along with the damping coefficient $(c)$ increasing, the curve of time deformation is a slowly decreasing trend.

Figure 5 shows the deformation of the beam by the mass of rail. Compared with copper and aluminum rail, the curve of time deformation has no significant changes. 


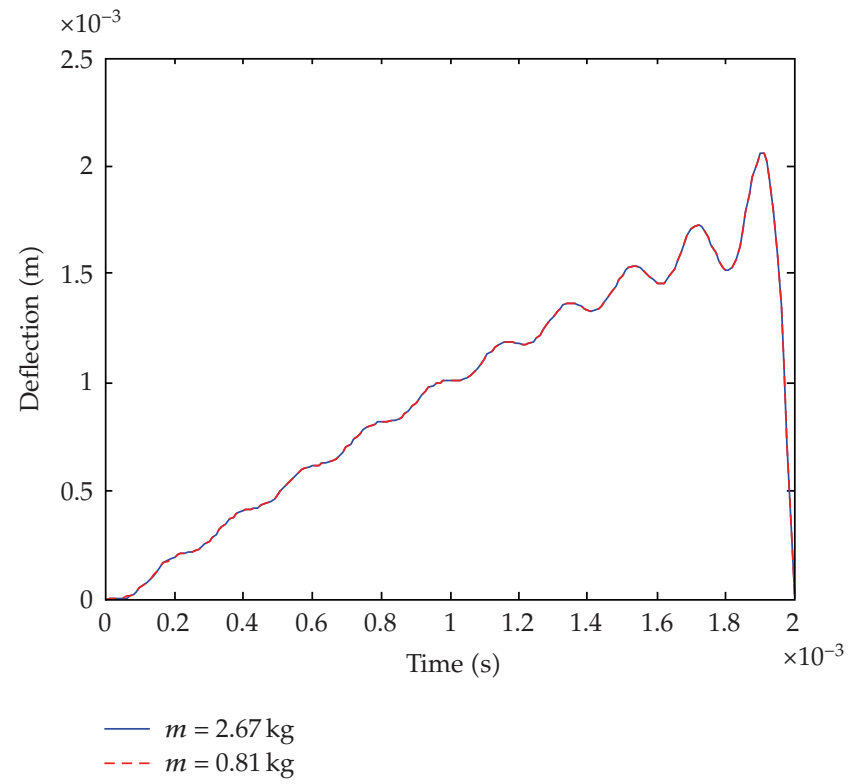

Figure 5: Deformation curve by differences mass of rail.

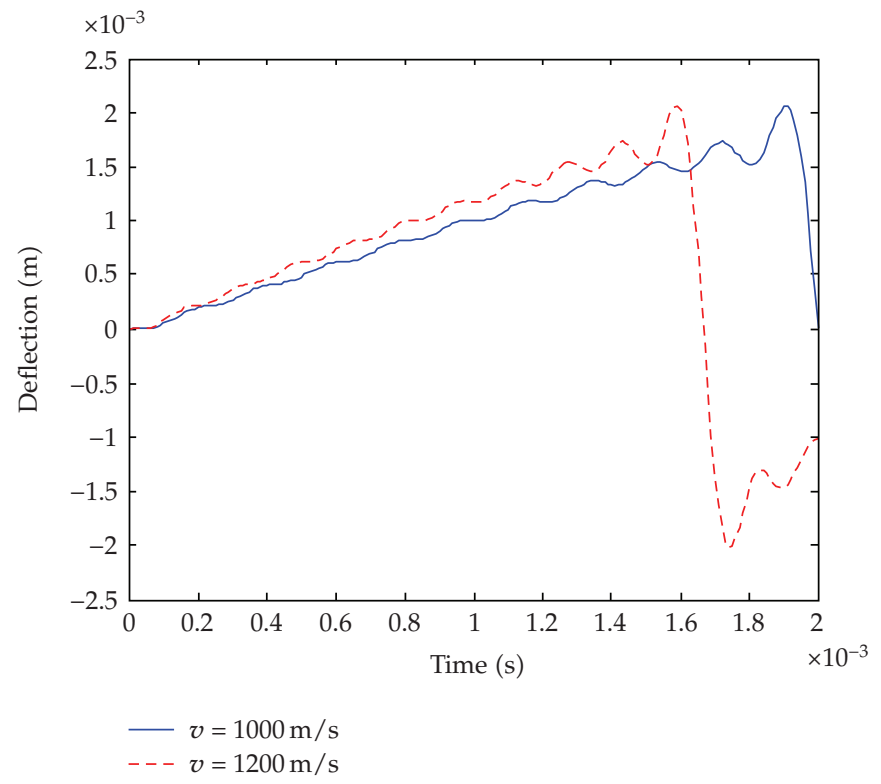

Figure 6: Deformation curve by differences velocity of the load.

Figure 6 shows the deformation of the beam by the load's velocity. Along with the load's velocity $(v)$ increasing, the curve of time deformation is an increasing trend. Under the calculating conditions given by this paper, for the rail of which $v$ equals to $1000 \mathrm{~m} / \mathrm{s}$, the deformation $(w)$ of the beam is $1.5 \times 10^{-3} \mathrm{~m}$ when the armature moves to the moment $t=1.6 \times 10^{-3} \mathrm{~s}$. While for the rail of which $v$ equals to $1200 \mathrm{~m} / \mathrm{s}$, the deformation $(w)$ of the 
beam is $2.05 \times 10^{-3} \mathrm{~m}$ at the same moment, and we can see that the former is $27 \%$ smaller than the latter.

\section{Conclusions}

(1) Taking the rail as a simply support beam on the elastic foundation and considering the damping force, a mechanical model for the electromagnetic railgun is built.

"We do not have general solution to nonlinear problems and some particular solutions are as few as treasures in history" [19]. In this paper, making use of variable method and the Lagrange equation including the damping force, the general solution of the homogeneous part, and the analytical solution of the governing equation subjected to sinusoidal pressures is derived which enriched and developed the theory of elastic mechanics with the hope to lay the foundation for solving the difficulty problem of electromagnetic rail subjected to arbitrary distribution function pressures.

(2) The deformation of beam which is influenced by the elastic coefficient, the damping coefficient, the mass of rail, and the load's velocity is analyzed by the MATLAB software. When the elastic coefficient is larger, the deformation of beam is smaller; when the load's velocity is larger, the deformation of beam is larger, the damping coefficient and the mass of rail have not obvious effect on the deformation of the beam.

\section{Acknowledgment}

The project support by the National Nature Science Foundation of China under (Grant no. 50875230).

\section{References}

[1] L. Liyi and L. Xiaopeng, "History and development trend of electromagnetic launch (EML)," Micromotors Servo Technique, vol. 37, no. 1, pp. 41-44, 2004.

[2] H. D. Fair, "Progress in electromagnetic launch science and technology," IEEE Transactions on Magnetics, vol. 43, no. 1, pp. 93-98, 2007.

[3] W. Ying and X. Feng, Principle of Electricgun, National Defense Industry Press, 1994.

[4] A. N. Smith, R. L. Ellis, J. S. Bernardes, and A. E. Zielinski, "Thermal management and resistive rail heating of a large-scale naval electromagnetic launcher," IEEE Transactions on Magnetics, vol. 41, no. 1, pp. 235-240, 2005.

[5] J. T. Tzeng and W. Sun, "Dynamic response of cantilevered rail guns attributed to projectile/gun interaction-theory," IEEE Transactions on Magnetics, vol. 43, no. 1, pp. 207-213, 2007.

[6] H. Yuwei, Modeling and Simulation of Electromagnetic Railgun System, Harbin Institute of Technology, Harbin, China, 2007.

[7] W. Sheng, Displacement field due to a moving load on Euler beam resting on an elastic half-space, M.S. thesis, Harbin Institute of Technology, Harbin, China, 2007.

[8] W. Liu and R. Shan, "Mathematic model and analytic solution for a cylinder subject to exponential function," Chinese Journal of Mechanical Engineering, vol. 22, no. 4, pp. 587-593, 2009.

[9] W. Liu, "Mathematical model and analytical solution for cylinder subject to uneven pressures," Chinese Journal of Mechanical Engineering, vol. 19, no. 4, pp. 574-578, 2006.

[10] S. Timoshenko, Mechanics of Material, Science press, Beijing, China, 1965.

[11] Y. Yanli, The research of the dynamic response of the rail system and viaduct by moving load, M.S. thesis, Wuhan University of Technology, 2002.

[12] J. T. Tzeng, "Structural mechanics for electromagnetic railguns," IEEE Transactions on Magnetics, vol. 41, no. 1, pp. 246-250, 2005. 
[13] Z. Shijian and L. Lingjun, Vibration Theory and Vibration Isolation, National Defense Industry Press, 2006.

[14] Z. Xiangting and W. Zhipei, Structure Vibration Mechanics, TongJi University Press, 2006.

[15] J. Shangnian and M. Yongli, Theoretical Mechanics, Higher Education Press, 2002.

[16] L. Ping and Z. Qingyuan, "Finite element analysis of infinitely long beam resting on continuous viscoelastic foundation subjected to moving loads," Journal of Traffic and Tran sportation Engineering, vol. 3, pp. 1-6, 2003.

[17] J. T. Tzeng, "Dynamic response of electromagnetic railgun due to projectile movement," IEEE Transactions on Magnetics, vol. 39, no. 1, pp. 472-475, 2003.

[18] A. J. Johnson and F. C. Moon, "Elastic waves and solid armature contact pressure in electromagnetic launchers," IEEE Transactions on Magnetics, vol. 42, no. 3, pp. 422-429, 2006.

[19] Z. Zhemin, Corpus, Science Press, Beijing, China, 1994. 


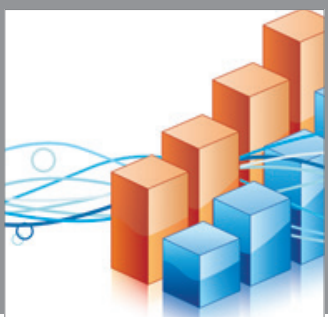

Advances in

Operations Research

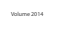

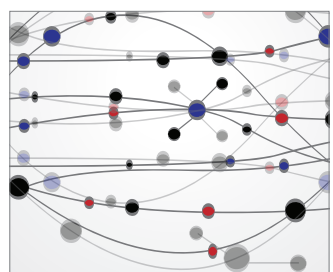

\section{The Scientific} World Journal
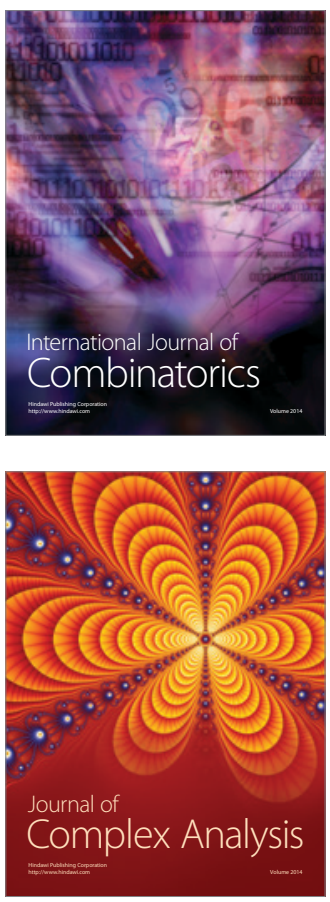

International Journal of

Mathematics and

Mathematical

Sciences
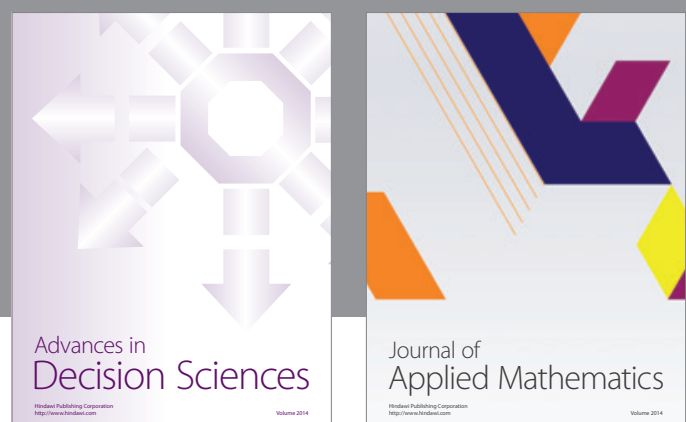

Journal of

Applied Mathematics
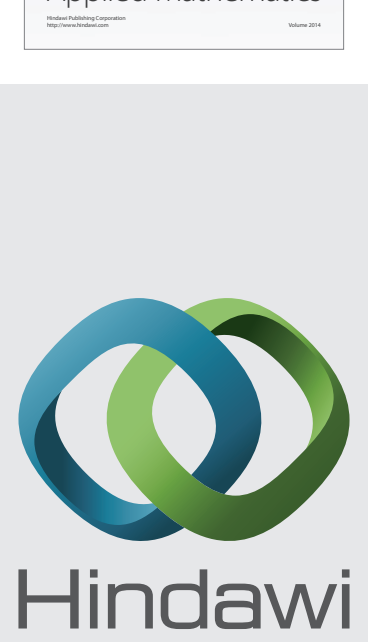

Submit your manuscripts at http://www.hindawi.com
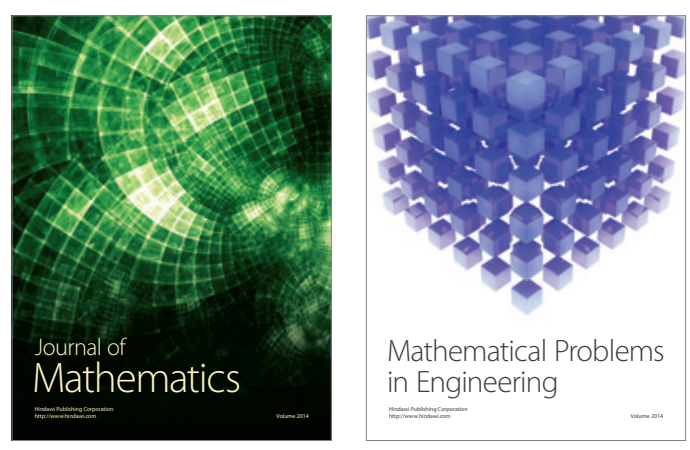

Mathematical Problems in Engineering
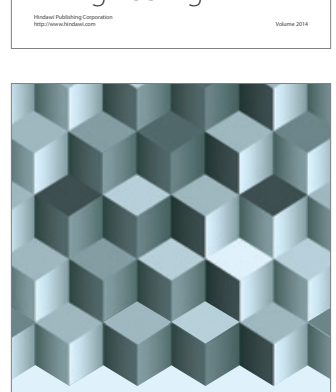

Journal of

Function Spaces
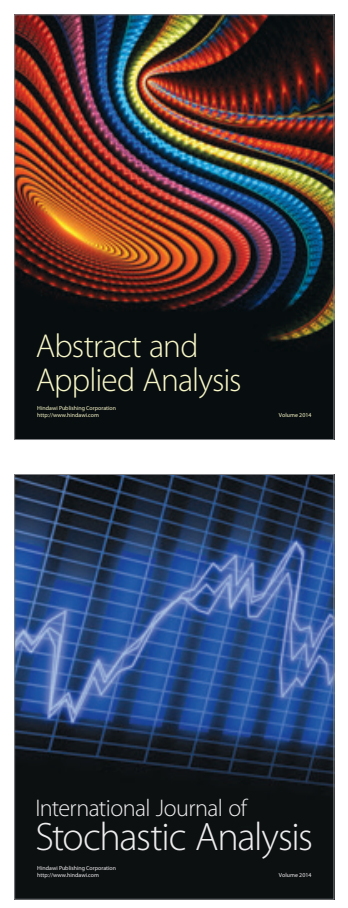

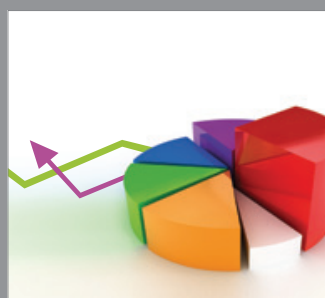

ournal of

Probability and Statistics

Promensencen
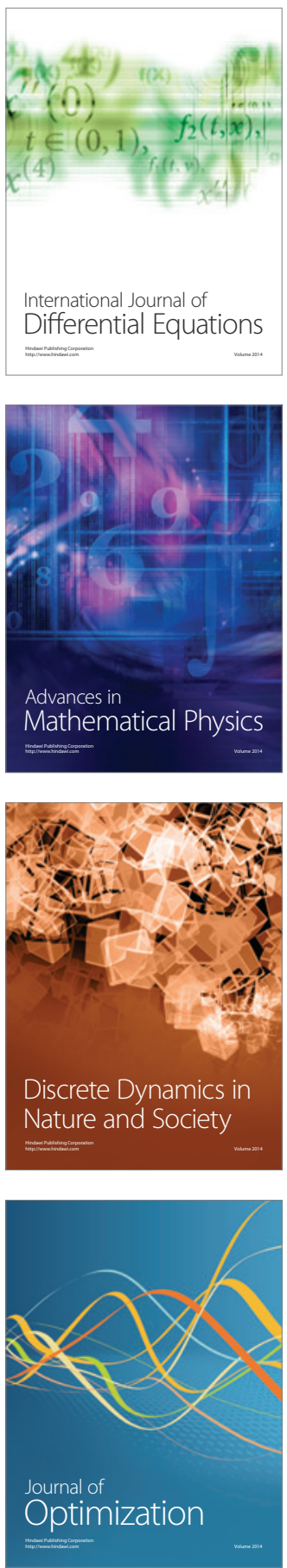